\title{
Challenges Facing the Application of Therapeutic Menus at Fayoum Public Hospital
}

Eman Ahmed ${ }^{* a}$, Mahmoud Hewedi ${ }^{\mathrm{a}}$, Naglaa A. El-Sherbiny ${ }^{\mathrm{b}}$, Omar Qoura ${ }^{\mathrm{a}}$

a faculy of Tourism and Hotels, Fayoum University, Egypt

${ }^{b}$ Faculty of Medicine, Fayoum University, Egypt

\section{ARTICLE INFO}

Keywords:

Therapeutic Menu

Hospital Food

Patient Meal

Nutritional Care

Challenges.

\begin{abstract}
Menus are considered the most critical tool in food and beverage services that determine the complete food operation i.e., the process, food labor, equipment, and costs. It is an integral part of nutritional care that allows patients to have a lot of choices enhancing their satisfaction with an adequate intake of nutrients required for a patient's speedy recovery. This study aims to identify obstacles and challenges facing nutritional care providers to ensure adequate application of therapeutic menus at Fayoum Public hospital Egypt. A descriptive qualitative case study was conducted on health care workers and food and nutritional care providers at Fayoum Public hospital using semi-structured interviews. Seven themes are reflecting the challenges of therapeutic menu application at Fayoum Public hospital. They are as follows: lack of clear policy and definite responsibilities, absence of patient involvement in the nutritional plan, limited food resources, neglecting the importance of nutrition care, insufficient competence of nutritional care providers, rigid menus within the solid hospital system, and deficient quality and inappropriateness meal. The results imply that the food and nutritional care system have their limits within hospital structure, resources, and organizations. Also, the importance of food and nutritional care plays a critical role in the application of the therapeutic menu. As quality food and nutrition care can improve patient outcomes and reduce costs leading to patient satisfaction
\end{abstract}

\section{Introduction}

Therapeutic diets are an integral part of hospital clinical treatment, needed for recovery from illnesses, injuries, etc. It can help to prevent malnutrition, restoring body tissues, and boost energy and immunity (Ghosh et al., 2019). Adding more hospitality to food service at hospitals by delivering a good quality nutritious meal contributes to patient satisfaction during his stay in the hospital. Patient satisfaction enhances food intake which is directly linked to the prevention of disease complications, quality outcomes, and cost reduction (Fernando and Wijesingheet al., 2017). Nutritional care is a fundamental aspect of the prevention and management of diseases, designed to improve the consistency and individualized care of the patient. It is referred to any practices conducted by nurses or dietitians to support a

\footnotetext{
*Contact Eman Ahmed at: eman_8287@yahoo.com
} 
patient to improve his dietary lifestyle. It also includes patient nutritional assessment, screening, evaluating, recognizing patients at risk of malnutrition, patient communication, and assistance (Ball et al., 2015).

Quality standards of food and nutritional care in public hospitals are a critical component of quality care and may improve patient recovery and wellbeing. Some considered nutrition care as the most effective factor to patient satisfaction; others suggested that meal quality is the most important factor affecting patient satisfaction. (Heede et al., 2020).

\section{Figure 1}

Hospital menus importance as a part of the food and nutritional care system

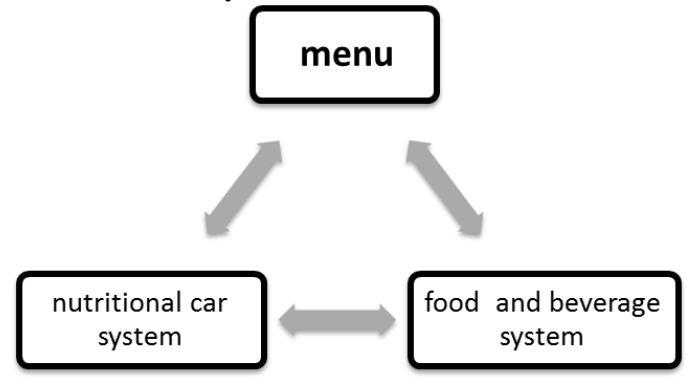

The National Health Services (NHS) developed standards for food, fluid, and nutrition care. These standards determine the minimum level of accepted performance with safe and effective health care services. Despite these standards, there is still a gap in the nutrition care of food at hospitals especially to patients at public hospitals in developing countries that receive poor quality meals of preparation and presentation (Safarian et al., 2019).

\section{Literature review}

The importance of hospital food and using food as medicine are not new; they can be traced back to one of the earliest medical works (Food is medicine- let your medicine be your food. (Hippocrates, 400 BC; Barker et al., 2011).

Malnourished hospital patients are exposed to higher complication rates (30.6 vs. $11.3 \%$ ) and three times greater risk of infection. Some studies reported higher mortality rates between malnourished patients compared to well-nourished patients (12 vs. 1\%). (Brotherton and Simmonds, 2010).

Understanding the importance of hospital therapeutic food and nutritional care service is a common problem all over the world. As a result, there is a need for more research that proves the importance of therapeutic diet in daily medical practices and care with diverse patient's diseases (Ghosh et al, 2019).

A therapeutic diet is an essential concept in health care and disease. It is a food that has its nutrients modified to meet the nutritional needs of a person and which forms a part of medical treatments to prevent symptoms or to improve nutrition state. Therapeutic diet plays a very crucial role in controlling and managing various diseases especially lifestyle diseases like diabetes, card vascular disease, and obesity (The Scottish Government. 2008).

Papier et al., (2017) mentioned that although the therapeutic diet is considered an integral part of medical care, there are many challenges against achieving well-established standards of care. It could be the absence of one or more of the following parameters: medical and nursing staff nutrition knowledge, awareness of the therapeutic menus, the existence of adequate nutrition care plan guidelines, nutrition care documentation, the availability of appealing food, and patient assistance with eating and drinking (Larsen et al., 2007; Mowe et al., 2008; Persenius et al., 2008; Papier et al., 2017).

Hospital menus are the most critical tool in the food service operation system as they determine operations, food labor, equipment, and other costs. They, also, determine the success of the whole process (Grigs, 2016).

Hospital menus are an integral part of nutritional care given that patient satisfaction with menu items enhances food intake of required nutrients for patient speed recovery and the prevention of disease complications reducing the hospital stay and cost (Grigs, 2016).

Providing hospital menus that cover all dietary and nutritional requirements in a hospital is a challenging task. There are many different types of patients and treatments that need to be met on an individual basis (Johns Hopkins Program for International Education in Gynecology and Obstetrics, 2016).

In 2008, Traner study concluded that menus at hospitals face some challenges such as staff time shortage, lack of clear and specific regulations for the menu variety and portion size, and lack of standard criteria for the assessment of the quality of menu nutrients and components (Wright, et al., 2011; Grigs, 2016). 
The food service system is a vital part of hospital operation, budget, and floor space. As a result, it should be operated in an optimal way (Olnay, 2003). Hospital food service, also, is a fundamental part of patient care given that a good nutritious meal contributes to patient recovery and prevents disease complications, and reduces costs (Greig, 2016).

However, studies have reported that public hospitals food service is suffering from poor quality of preparation and presentation in developing countries (Hartwell, et al., 2004).

Maintaining the meal appearance palatability and nutrition content together is considered as an indicator of food quality service. The meal appearance palatability and nutrition content together are considered also as a substantial challenge to any catering institution (Hartwell, 2004).

Stanga et al., (2003) reported that Meal temperature and texture are considered the most effective aspect of quality catering service. Meal temperature is considered an area of patient dissatisfaction and complaint (Necolien, 2019).

Many studies examined the relationship between service quality, food quality, and customer satisfaction and the results have confirmed a significant relationship between these constraints (Al-Tit, 2015; Torky, et al., 2015). Failure to provide food appropriately to the patient is considered one of the major causes of undernutrition in hospitals (Nicolien, 2019). Also, the poor presentation of food due to the length of meal transportation, inadequate meal temperature, and texture are common barriers to hospital quality food service at hospitals.

Nutrition care refers to any practice conducted by dieticians or nurses to improve or support their dietary behavior (Ball et al., 2015). Nutrition care may include any aspects of nutrition assessment, nutrition advice or counseling, nutrition screening, and nutrition evaluation. Adequate food and nutrition care service could improve the quality of hospital care and improve patient clinical outcomes. Promoting optimal nutrition care through quality food service can lead to faster healing and decreasing the length of hospital stay, which has a significant impact on hospital costs (Kandrap, 2004; Moghaddasi, 2019).

Irina Papier et al, (2018) concluded that the main challenges of optimal nutrition care are the lack of available nutritional therapy protocols, dietitians, the time it takes to identify patients in need of nutrition therapy, and the lack of staff awareness of the importance of identifying patient's nutritional needs. The good thing is that all these previous factors are manageable when nutritional care becomes a priority with a clear nutrition policy and the collaboration of medicals, nurses, and authorities to establish and implement patient's center care policy (Bruno et al., 2013; Lotfi et al., 2019)

The provision of right and adequate nutrition and hydration is a human right and a significant requirement in the delivery of health care. The consequences of hospital food borne infections range from patient annoying to patient lifethreatening which can lead to death in the vulnerable group (Mentziou et al., 2014).

Hospital policies aim to provide a framework for the staff explaining how the hospital will ensure the appropriate food fluid and nutrition care will be available to meet the individual patient's needs. Procedures aim to clearly define the policy determining the sequence of steps that staff have to follow to meet the organization's goals and to ensure the quality of provided service (Tappenton et al., 2013).

Health care service is a complex mixture of responsibilities that requires a high level of knowledge and skills. It includes different and variable clinical decisions that are related to patient health (Johns Hopkins Program for International Education in Gynecology and Obstetrics, 2010). However, most of the medical and nursing staff lacks adequate nutrition knowledge to address diet and nutrition-related issues with their patients (Yasuoka et al., 2016).

Also, nutrition and dietary knowledge are not a requirement for anyone involved in preparing food for public health services (Hartwell, 2004). Such nutrition knowledge deficiency results in poor nutrition competence for health workers (Mowe et al., 2008).

\section{Methodology}

A descriptive qualitative case study was conducted at Fayoum public hospital during the summer of 2019. A purposive heterogeneous sampling technique was used. Seventeen health care workers including the general manager of the hospital, infection control and quality department manager, one head nurse, and fourteen nurses from different wards were interviewed. An interview was 
conducted with fourteen food and nutritional care providers including one dietitian, one cooker, seven for food preparation and meal backing, two for food delivery, and three for cleaning workers. The semi-structured interviews questions were designed based on national health service (NHS) food, fluid, and nutrition care standards set by the Scottish government as the minimum level of accepted service quality which demonstrates delivery of safe, effective, and person-center healthcare and understands and promote the care improvement. The researcher used these standards as a tool to assess the quality of food and nutrition care service and determine the main challenges and obstacles facing the food and nutrition care system at Fayoum public health hospital in Egypt.

The semi-structured interviews were in four forms:

1. The first interview is for the hospital manager and the head of the quality and infection control department.

2. The second interview was designed to obtain data from the hospital dietitian and the nutrition inspector from the governance of the health affairs directorate.

3. The third interview was for the kitchen staff of the hospital.

4. The fourth interview was conducted with the head nurse and the head nurses of the different hospital wards.

A pilot study was conducted at the Fayoum University Teaching Hospital in 2018 to test the questionnaire form regarding clarity and simplicity, estimate the time needed to complete the interview, have a look at the workflow. Few modifications were done for some confusing or unapplicable questions.

Official permission was obtained from the Fayoum Health Directorate and the general manager of the Fayoum Public hospital. Informed consent was obtained from participants with the assurance of data confidentiality before they were enrolled in the study.

The mean and standard deviation (SD) of age were calculated. Sex, years of experience, and occupation of study participants were presented as frequencies. The collected data were analyzed by qualitative inductive approach with a framework type analysis. First by coding the data then interpret the meaning of each code so that the text meaning can be seen more clearly to develop the main themes. Nurse evaluation for meal quality was presented as mean.
In Egypt, all the public hospitals are following the same food menu, portion size, and mealtime regardless of individual patients' needs. The study was conducted at one of the large public hospitals in Fayoum Governorate in Egypt. Fayoum Governorate is one of twenty-seven governorates in Egypt with a total population of 3.359.399 million. Fayoum public hospital is the biggest referral hospital in Fayoum out of six Public hospitals. Most residents in the governorate live in rural areas, with an urbanization rate of $22.5 \%$. Therefore, the hospital is responsible for a heterogenic population differing with demographic characteristics as $26.3 \%$ of the population suffers from poverty and $28 \%$ are illiterate (CAPMAS, 2016). 4 .

\section{Results}

The characteristics of the study group were shown in the following table.

\section{Table 1}

Characteristics of the Health Care Workers \& Food Handlers

\begin{tabular}{|c|c|}
\hline Variables & $\mathbf{N}$ \\
\hline \multicolumn{2}{|l|}{ Age } \\
\hline Mean \pm SD & $41 \pm 6$ \\
\hline Range & $23-56$ \\
\hline \multicolumn{2}{|l|}{ Sex } \\
\hline Male & 5 \\
\hline Female & 27 \\
\hline \multicolumn{2}{|l|}{$\begin{array}{r}\text { Years of experience } \\
\end{array}$} \\
\hline Less than 5 years & 5 \\
\hline More than 5 years & 27 \\
\hline \multicolumn{2}{|l|}{$\begin{array}{r}\text { Study participants } \\
\end{array}$} \\
\hline Health care workers & 17 \\
\hline Hospital Manger & 1 \\
\hline Infection control and Quality Manger & 1 \\
\hline Head nurse & 1 \\
\hline Nurses & 14 \\
\hline Nutrition \& food handlers & 15 \\
\hline Nutrition inspector & 1 \\
\hline Dietitian & 1 \\
\hline Kitchen staff & 13 \\
\hline
\end{tabular}

Regarding the National Health Services (NHS), Food and Fluid Nutritional Care Standards, the study results present seven main themes that reflect the challenges at the hospital. They are as follows: lack of clear policy and definite responsibilities, neglecting the importance of nutrition care, insufficient competence of nutritional care providers, rigid menus within the solid hospital 
system, absence of patient involvement in the nutritional plan, limited food resources, and inappropriateness meal.

The following figure illustrates the main challenges and obstacles against adequate food and nutrition care service at Fayoum public health hospital.

\section{Figure 2}

Challenges and obstacles facing food and nutrition care at Fayoum Public Hospital

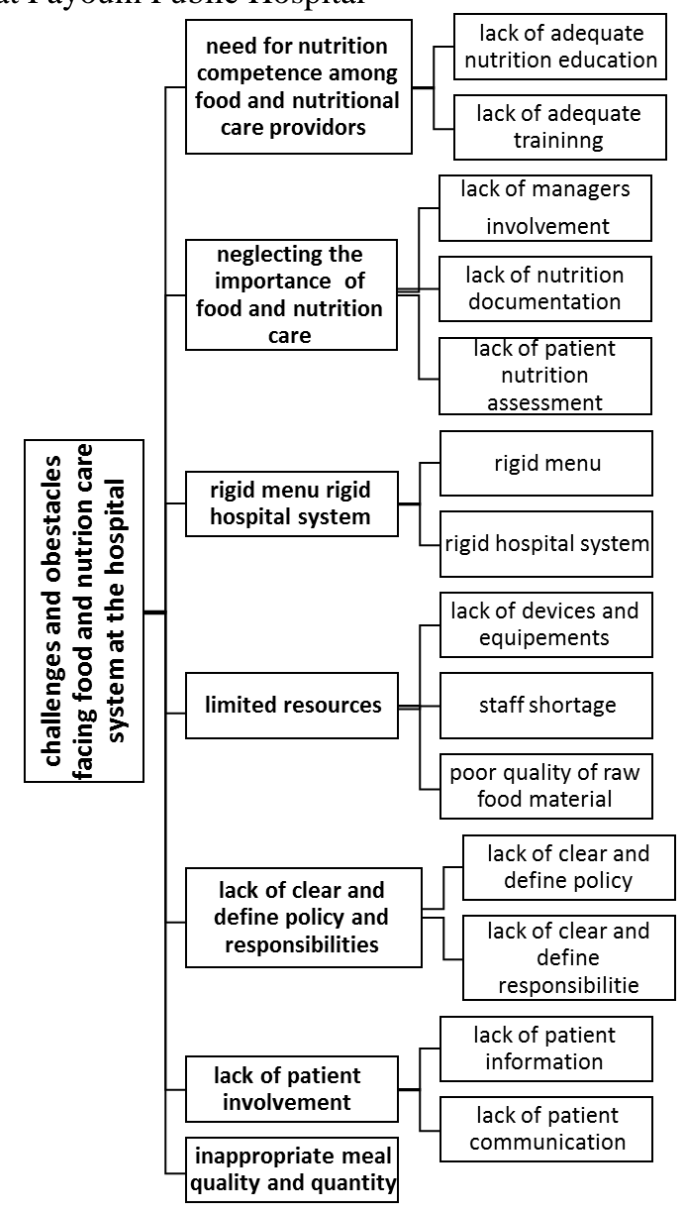

4.1. Lack of clear policy and definite responsibilities

Regarding the NHS standard criteria for hospital policy of food services, to be formally defined and contain a clear and definite responsibility of the food and nutritional care staff. The study results revealed that Fayoum Public hospital food service policy is lacking the main steps to be followed as a constant and repeated approach to ensure safe efficient and quality meal preparation with no clearly defined responsibilities of the staff as handling raw food material, cleaning schedules, infection control and dealing with injures and wounds during work. As well, the nutritional care sheet is not documented and it did not contain patient nutritional assessment and monitoring tools, to recognize and treat a patient at risk of malnutrition. The hospital food policy is updated every two years, and the reports of food and nutritional care problem reports are not a part of the updating process as mentioned by the head of the infection control unit in the hospital (policymaker). Examples of the words by the hospital staff related to the clear policy theme. The response cited as follows:

Participant 8, kitchen staff: "I learned my duties from those who worked before me."

Participants $5 \& 10$ nurses: "I think to improve the food and nutritional care system; we need clearer and define policy and procedures.

\subsubsection{Neglecting the importance of food and nutrition care}

The hospital dietitian and most of the nurses noted that food and nutrition care is not a priority for the health care professionals. Also, the head nurse stated that in most cases food and nutrition care are not essential factors for patients unless their medical conditions were associated with nutritional problems. For example, a blood test for anemia is mainly taken to check the need for blood transfusion not for insuring adequate nutritional care. As cited by the hospital manager who said: "I only approve the food and nutrition policy established by the infection control unit in the hospital." The head nurse reported a lack of a documented system for food and nutrition care. "We only document the type of meal needed for a patient medical condition."

\subsubsection{Insufficient competence of nutritional care providers}

The study results appeared that some essential terms like nutritional assessment, monitoring, evaluating, and recognizing patient with risk of malnutrition seem to be unfamiliar among most of the nurses which are consistent with what the head nurse mentioned: "We do not have nutrition assessment tools to recognize and treat a patient at risk of malnutrition and the nurses have never had any training about it".

They seem to lack skills for individualizing nutrition care needs as they said for example:

(5 Participant, nurse 10): "If a patient asks for help, I provide it" she didn't have sufficient information that she was supposed to assess the patient's need 
for assistance instead of waiting for the patient to ask.

Also, most of the nurses complained about meal preparation and presentation referring to the necessity of raising the kitchen staff competence to improve food service at the hospital. The kitchen staff seems to lack the basic information about nutrition and food service and skills for food preparation and presentation as their educational level does not exceed technical education and even some of them are illiterate. They did not receive any nutritional training that qualifies them to prepare and deliver safe and effective meals. The only training they have ever had is infection control procedure in dealing with food. (Participant 8, nurse): "The meal presentation is inferior". (Participant 4, nurse): "I think the food service system at hospitals should be improved through more competent kitchen staff."

\subsubsection{The rigid menu within the solid hospital system}

Hospitals were restricted to changing menu, portions, or mealtime, which gives the hospital limited opportunities to individualize food and nutritional care.

The hospital manager and dietitian described that the low flexibility of the food and nutrition care system gave them limited opportunities to individualize nutrition care.

The hospital manager: "The food and nutrition care policy is limited by specific terms of the Ministry of Health"

The hospital dietitian: "We are committed to a specific cycle menu even if it does not appeal to the patient. For example, if the patient refused a specific kind of meal, it isn't in our authority to change it."

Head nurse: "I think that one of the obstacles against patient involvement is the inflexible system."

\subsubsection{Absence of patients' involvement in the nutritional plan}

The food and nutrition care system at the hospital is not recognized by the patient such as the available facilities for eating, special equipment, assistance with eating and drinking, mealtimes, the availability of food out of mealtime, how to order missing meals. Also, the communication between the patient and the hospital nurses and dietitians does almost not exist as described by the hospital dietitian. And the role of the dietitian is only limited to kitchen supervision, without any contact with the patients. Some nurses justified the reason for this lack of communication with the patients is due to shortage of staff numbers and work overload while others reported that the inflexible system is a primary obstacle against individualized care. While others pointed to that the patient education level and nutrition awareness is an obstacle against patient involvement.

Participant 4, nurse: "Patient assistance with eating and handwashing returns to the person accompanying the patient."

The head nurse: "Patient assistance could only happen while undergraduate nurses are around."

Participant 10, nurse: "If a patient asks for help, I provide it."

Participant 4 nurse: "Often the patient does not depend on the provided meal by the hospital."

\subsubsection{Limited food resources:}

The kitchen staff expressed their urgent need for some new devices and equipment in the hospital kitchen. They were struggling with the existing resources because most of the devices and equipment were either worn out or need maintenance which is an obstacle facing the daily work. That makes it difficult for them to provide satisfactory food service at the hospital.

Participant 12, kitchen staff: "We need a new food heater because the one we have is broken."

Participant 4, kitchen staff: "We need a packing machine."

The hospital dietitian: "We need a new oven for chicken roasting because the one we have here is broken. We, also, need a freezer unit to store the raw food ingredient and any remains of food." "The devices and equipment we have here been around for the last twenty years and they are no longer suitable for work."

Also, the kitchen staff complains of the staff shortage, which makes them overloaded.

Furthermore, the nurses indicated a lack of resources through the need for hot and cold-water units to enable the patient to obtain drinks 24 hours a day, which is an essential need for patient hydration. As well, the poor quality of raw food materials is another obstacle as expressed by the dietitian and some participant nurses.

The hospital dietitian: "We use frozen meat and chicken which shrink after cooking and become difficult to chew, affecting the portion size that is supposed to be served to the patient." 
Participant nurses, 4,6, 8, 10 \& 11: "The presented meal is in poor quality and quantity and needs more improvement.

\subsubsection{Inappropriate meal quality}

The nurses evaluate the meal from one to five as five is very convenient to the patient and one is inconvenient according to their experience. The mean of their evaluation was as follows:

Table 2

Nurses' hospital meals evaluation

\begin{tabular}{|l|l|}
\hline Statement & mean \\
\hline Meal convenience to patient individual needs & 3 \\
\hline $\begin{array}{l}\text { Meal convenience to the patient clinical } \\
\text { condition }\end{array}$ & 3.1 \\
\hline $\begin{array}{l}\text { Meal temperature convenience at the service } \\
\text { time }\end{array}$ & 3.8 \\
\hline Meal texture convenience to patient needs & 3.3 \\
\hline Mealtime convenient to the patient's needs & 3.4 \\
\hline $\begin{array}{l}\text { Availability of meal equipment that is } \\
\text { convenient for the patient medical need }\end{array}$ & 2.75 \\
\hline
\end{tabular}

Some nurses mentioned that the meal portion size was insufficient, and the mealtime is not convenient especially the dinner which is served at $2 \mathrm{pm}$. and the next meal (breakfast) is at $8 \mathrm{am}$., which means the difference in time is about 18 hours not including any snacks between them. Also, most of the nurses complained about the meal appearance and backing as it is not convenient.

\section{Discussion}

The food service and nutrition care system at the hospital are facing many challenges and obstacles. Each patient had a basic right to have his fundamental needs to be fulfilled as food and nutrition care. There were some important elements in food services such as the communication of dietitians and nurses with patients, lack of patient involvement in food choices (meal choice, portion size, and mealtime), and nutrition care assessment and monitoring that may result in, inadequate food and nutrition care which may lead to malnutrition, complications of the disease and poor patient outcomes.

The kitchen staff and nurses in the study described some challenges and obstacles more dependent on hospital structure and limited food and nutrition care resources, incompetent of the food and nutrition care providers, a need for clear and definite policy and responsibilities.

\subsection{Clear and define food and nutrition policy and responsibilities}

Nutrition care is a multidisciplinary field involving several professions in hospitals, having a clear policy helps to ensure high-quality service meeting the organization's objectives (Dahi et al., 2014). Food and nutritional care providers should play an active role as a part of the team, with clearly defined responsibilities and promotions of engagement with patients (Marshall et al., 2019).

The study results showed that a lack of food and nutrition care policy and procedures including the responsibilities of food and nutrition care providers result in no accountability of patient nutrition. Therefore, malnutrition of patients is more likely to happen without being recognized or treated.

\subsection{Patient involvement and communication with competent food and nutrition care providers:}

In the current study, patient information and communication seem to be almost missing with dietitians. In contrast (the European Federation of the Associate Dietitian, 2017) stated that information and communication about food and nutrition care are delivered to the patient in a suitable format to prevent unmet nutritional needs of the patients due to poor communication.

The kitchen staff at the hospital in this study seems to be having insufficient knowledge of food and nutritional care service. As the kitchen staff education level doesn't exceed the technical education with no nutritional background on food service that qualifies them to prepare a safe and effective meal and fulfill the nutritional needs of the patients, which affect patient food intake and satisfaction.

Dietitians play a crucial role in health services to reduce malnutrition forms, change eating behavior and promote a healthy lifestyle (Ball et al., 2015). The Dietitian's role is to translate medical and nutrition patient needs into food preparation and ensure that the patient receives a meal suitable for his medical condition and nutrition needs in an optimal condition (Orit Yona et al., 2020).

\subsection{Hospital management and food and nutrition care system}

From another perspective, the nurses and dietitians in this study expressed frustration regarding the rigid nutrition care system and the lack of manager involvement. The manager should consider that food and nutrition care services play an essential role in the medical treatment service provided to the patient. Also, they ought to define clearly what the food service and nutrition care service should 
include and give primary attention to food and nutrition care policy and procedures.

As Tappenden et al., (2013) noted patient center care means that food and nutrition care services are responsive to individual needs, which includes a flexible system that allows for individualization. Clinical and administrators often should realize the importance of food and nutrition care, understanding the extent of malnutrition in their institution and its potential impact on costs and or quality.

\subsection{Therapeutic menu and patient satisfaction}

Finally, the current study noticed that public hospitals all over the country use the same menus, which is recommended by the Ministry of Health regardless of the demographic data and food culture.

The menu is the most critical tool in food service because it determines food labor, equipment and other costs, and the success of the whole food service operations. Various food service interventions such as assisting patients during meals and encouraging patients to choose food may improve patient outcome measures and patient satisfaction (Nicolian et al., 2019). Patient satisfaction with menu items enhances food and nutrition intake, which is needed for patient recovery (Grigs et al., 2016). However, providing patient center-nutrition care could be challenging for health care professionals due to the complicity of patient nutrition problems and the organization of constraints (Hestevik et al., 2019).

Improper food service performance, low human resources and sanitation, absence of adequate nutritional care training, and lack of awareness of patients' expectations are considered the main challenges against quality food and nutritional care service at Menoufya Teaching Hospital in Egypt (Badr et al., 2019). These results should be a trigger for a big, planned project to improve food and nutrition care service at the hospitals in Egypt as a part of health care quality improvement.

Patient satisfaction is considered as a critical factor in determining the quality of health care service at hospitals and the need to assess food service at hospitals is not only important for patient nutrition and welfare, but it also has financial benefits as decreasing food wastage, staff time, and length of hospital stay, which helps hospitals gain a good reputation (Abdelhafez et al., 2012).

\section{Conclusions and Recommendations}

This study provides information about challenges and obstacles facing the whole food and nutritional service at Fayoum Public Hospital. Seven main themes reflect the challenges of the therapeutic menu at the hospital. They are as follows: lack of clear policy and definite responsibilities, absence of patient involvement in the nutritional plan, limited food resources, neglecting the importance of nutrition care, insufficient competence of nutritional care providers, the rigid menus within the solid hospital system, and deficient quality and inappropriateness meal. The results imply that the food and nutritional care system have their limits within hospital structure, resources, and organizations. Also, the importance of food and nutritional care plays a critical role in the application of the therapeutic menu. Quality food and nutrition care can improve patient outcomes and reduce costs leading to patient satisfaction.

The research offered a set of recommendations for the ministry of health, hospital management, kitchen staff, and quality control management

Recommendations for the Ministry of Health

1. Set a manual for food and nutritional care service to follow service quality.

2. Using nutrition assessment tools to determine and deal with the malnourished patient.

3. Carrying out awareness campaigns for individuals on the importance of good nutrition for a better life

\section{Recommendations for The Hospital Managers}

1. Continuous training programs for health care providers especially the nurses and the kitchen staff.

2. Monitoring patients' nutritional status during the hospital stay to determine patients at risk of malnutrition and its potential impact on costs and quality.

3. Giving more attention to patients' complaints, and patient satisfaction.

Recommendations for the Kitchen staff

1. Good presentation of the meal to allow them to consume it.

2. Training of cooking and serving meals in meals preparation, and packing for the kitchen staff to ensure the quality of food service.

Recommendations for Hospital Quality and Infection Control Department

1. The hospital food and nutrition care policy should be patient-focused to ensure the 
delivery of safe meal that meets individual nutritional needs.

2. The hospital menus should be regularly reviewed and consider religious and cultural patterns and the need for equality of access.

In qualitative research, the goal is to enhance the understanding of the phenomenon being studied. The results from this study can't be generalized, but they may have transferability to a similar sample context. The size of the sample is small, and all the participants are from the same hospital; however, the hospital is extensive and provides health care services for more than three million people. The challenges and obstacles identified reflect the point of view of the hospital staff and researcher.

\section{References}

Abdelhafez, A. M., Al Qurashi, L., Al Ziyadi, R., Kuwair, A., Shobki, M., \& n Mograbi, H. (2012). Analysis of factors affecting the satisfaction levels of patients toward food services at general hospitals in Makkah, Saudi Arabia. Age (years), 30, 31-50.

Al-Tit, A. A. (2015). The effect of service and food quality on customer satisfaction and hence customer retention. Asian Social Science, 11(23), 129.

Al-Torky, M. A., Mohamed, E. A., Yousef, F. M., \& Ali, N. A. (2016). Inpatients' satisfaction with food services in Sohag University Hospital. The Egyptian Journal of Community Medicine, 34(2), 33-45.

Badr, S., Shaheen, H., El Sayed, M. E., \& Basiony, L. A. (2019). Gap analysis of patients 'nutritional care and food services at menoufia university hospitals.

Ball, L., Leveritt, M., Cass, S., \& Chaboyer, W. (2015). Effect of nutrition care provided by primary health professionals on adults' dietary behaviours: a systematic review. Family Practice, 32(6), 605617.

Barker, L. A., Gout, B. S., \& Crowe, T. C. (2011). Hospital malnutrition: prevalence, identification and impact on patients and the healthcare system. International journal of environmental research and public health, 8(2), 514-527.

Brotherton, A., Simmonds, N., \& Stroud, M. (2010). Malnutrition matters. In Meeting Quality Standards in Nutritional Care. BAPEN.

Bruno F. Sunguya, Krishna C. Poudel, Linda B. Mlunde, David P. Urassa, JunkoYasuoka and Masamine Jimb. (2013). 'Nutrition training improves health workers' nutrition knowledge and competence to manage child undernutrition: a systematic review'. Frontiers in Public Health, 1(37).

Central Agency for Public Mobilization and Statistics (CAPMAS) Egypt, (2016). URL: https://www.capmas.gov.eg/ [retrieved 2019-1007].

Dahl Eide, H., Halvorsen, K., \& Almendingen, K. (2014). Barriers to nutritional care for undernourished hospitalised older people. Journal of Clinical Nursing, 24(5-6), 696-706.

Dorian Nicolien Dijxhoorn. (2019). 'HOSPITAL MEAL SERVICES'. Online thesis.

El-Sherbiny. N. A., et al. (2017). Patients' Satisfaction with Delivered Food Services in Fayoum Hospitals. EC Nutrition 9.2 94-104.

European federation of the association of dietitian, (2017). The role of European public health dietitians. The Voice of European Dietitians: www.efad.org

Fernando GHS, Wijesinghe CJ. (2017). Quality and standards of hospital food service; a critical analysis and suggestions for improvements. Galle Medical Journal, 122(2), 17-21.

Ghosh, A., Paul, S. K., Pal, R., \& Pal, S. (2019). Therapeutic diet for primary care. Al Ameen Journal of Medical Sciences, 12(1), 37-43.

Greig, Susan, (2016). Hospital Menu Assessment of Nutrient Composition and Patient Satisfaction. Electronic Thesis and Dissertation Repository. 4373. https://ir.lib.uwo.ca/etd/4373.

Moghaddasi, H. (2019). The Importance of Nutrition Information System for Improving Patient Care. Acta Scientific Nutritional Health 3(4), 26-27.

Heede, K., Cornelis, J., Bouckaert, N., Bruyneel, L., Van de Voorde, C., \& Sermeus, W. (2020). Safe nurse staffing policies for hospitals in England, Ireland, California, Victoria and Queensland: A discussion paper. Health Policy, 124(10), 10641073.

Hestevik, C. H., Molin, M., Debesay, J., Bergland, A., \& Bye, A. (2019). Healthcare professionals' experiences of providing individualized nutritional care for Older People in hospital and home care: a qualitative study. BMC geriatrics, 19(1), 1-9.

Johns Hopkins Program for International Education in Gynecology and Obstetrics (ECOQUIP), (2016). Challenges of sustainable and healthy food for the healthcare sector.

Kondrup, J.; Johansen, N.; Plum, L.M.; Bak, L.; Larsen, I. H.; Martinsen. A (2002). Incidence of nutritional risk and causes of inadequate nutritional care in hospitals. Clin. Nutr, 21,461-468.

Lassen, K. O., Kruse, F., \& Bjerrum, M. (2005). Nutritional care of Danish medical inpatientspatients' perspectives. Scandinavian journal of caring sciences, 19(3), 259-267.

Lotfi, M., Zamanzadeh, V., Valizadeh, L., \& Khajehgoodari, M. (2019). Assessment of nursepatient communication and patient satisfaction from nursing care. Nursing open, 6(3), 1189-1196.

Marshall, A. P., Takefala, T., Williams, L. T., Spencer, A., Grealish, L., \& Roberts, S. (2019). Health 
practitioner practices and their influence on nutritional intake of hospitalised patients. International journal of nursing sciences, 6(2), 162168.

Mentziou, I., Delezos, C., Nestoridou, A., \& Boskou, G. (2014). Evaluation of food services by the patients in hospitals of Athens in Greece. Health Science Journal, 8(3), 383.

Mowe, M., Bosaeus, I., Rasmussen, H. H., Kondrup, J., Unosson, M., \& Irtun, Ø. (2006). Nutritional routines and attitudes among doctors and nurses in Scandinavia: a questionnaire based survey. Clinical Nutrition, 25(3), 524-532.

Orit Y., Rebecca Goldsmith and Ronit Endevelt. (2020). Improved meals service and reduced food waste and costs in medical institutions resulting from employment of a food service dietitian - a case study. Journal of Health Policy Research. 9(5), 19.

Papier, I., Lachter, J., Hyams, G., \& Chermesh, I. (2017). Nurse's perceptions of barriers to optimal nutritional therapy for hospitalized patients. Clinical nutrition ESPEN, 22, 92-96.

Safarian M, Alinezhad-Nameghi M, Vafisani F, Asadi Z, Seyedhamzeh Sh. (2019). Patient Satisfaction with Hospital Food in the Hospitals Affiliated to Mashhad University of Medical Sciences, Iran. J
Nutrition Fasting Health, 6(4): 191-197. DOI: 10.22038/JNFH.2019.32579.1141

Tappenden, K. A., Quatrara, B., Parkhurst, M. L., Malone, A. M., Fanjiang, G., \& Ziegler, T. R. (2013). Critical role of nutrition in improving quality of care: an interdisciplinary call to action to address adult hospital malnutrition. Journal of the Academy of Nutrition and Dietetics, 113(9), 12191237.

The Scottish Government. (2008). Food in hospitals: National catering and nutrition specification for food and fluid provision in hospitals in Scotland. Retrieved September 3, 2016 http://www.gov.scot/resource/doc/229423/006218 $\underline{5 . p d f}$

Theurer, V. A. (2011). Improving Patient Satisfaction in a Hospital Foodservice System Using Low-Cost Interventions: Determining Whether a Room Service System is the Next Step. All Graduate Plan B and other Reports. 32. https://digitalcommons.usu.edu/gradreports/32.

Wright, O., Capra, S., \& Aliakbari, J. (2003). A comparison of two measures of hospital foodservice satisfaction. Australian Health Review, 26(1), 7075.

Zolkef. Y. (2017). Evaluating the Concept of Choice in Healthcare. Special Communication, 24(6), 92-96 